\title{
Bilateral Spontaneous Rupture of the Achilles' Tendon During Chemotherapy Treatment
}

\author{
Bilge KESIKBURUN, ${ }^{1}$ Emel EKŞioĞLU, ${ }^{1}$ Alper DiLli, ${ }^{2}$ Aytül ÇAKCI ${ }^{1}$ \\ 'Department of Physical Medicine and Rehabilitation, Dışkapı Yıldırım Beyazıt Training and Research Hospital, Ankara-Turkey \\ ${ }^{2}$ Department of Radiology, Dışkapı Yıldırım Beyazıt Training and Research Hospital, Ankara-Turkey
}

\begin{abstract}
Achilles rupture can occur spontaneously or secondary to traumatic injury. Described in the present report is the case of a patient with lung cancer who was undergoing combined chemotherapy treatment that included corticosteroids. Diagnosis of bilateral spontaneous Achilles' tendon rupture was initially overlooked. A-60-year old male presented with complaints of intense bilateral ankle pain, weakness in the legs, difficulty walking on the toes, and swelling and bruising on the heel. Neuropathy associated with chemotherapeutic agents was considered, before physical examination and magnetic resonance imaging (MRI) revealed bilateral Achilles' tendon rupture. The complaints resolved, following a 4-week rehabilitation program. Spontaneous rupture of the Achilles' tendon may occur as a rare complication in patients taking chemotherapy agents including steroids.

Keywords: Achilles rupture; steroid; complication; chemotherapeutic agents.

Copyright $\odot$ 2016, Turkish Society for Radiation Oncology
\end{abstract}

\section{Introduction}

Spontaneous Achilles' tendon rupture is a rare condition that may occur as a complication of local injection, diabetes mellitus, systemic lupus erythematosus, rheumatoid arthritis, gout, obesity, or chronic renal failure.[1] Systemic steroid treatment is also associated with spontaneous Achilles' tendon rupture.[2] Presently described is the case of a patient with lung cancer who developed bilateral simultaneous and spontaneous Achilles' tendon rupture during combined chemotherapy treatment that included corticosteroids. The diagnosis was initially overlooked, and the patient was thought to have neuropathy associated with chemotherapeutic agents.

\section{Case Report}

A 60-year-old male presented with complaints of in- tense bilateral ankle pain, weakness in the legs, difficulty walking on the toes, and swelling and bruising on the heel. The patient described feelings of numbness and tingling in the feet and simultaneous abnormal sound in both ankles just before onset of symptoms. The patient had non-small-cell lung cancer and was undergoing a second cycle of chemotherapy, consisting of a monthly protocol including gemcitabine, cisplatin, vincristine, and systemic corticosteroid of $16 \mathrm{mgr}$ dexamethasone. The patient was initially thought to have neuropathy associated with the chemotherapeutic agents. No treatment was administered, and the chemotherapy protocol was continued.

After the ankle pain and difficulty walking persisted for 2 weeks, the patient was referred. On physical examination, both ankles appeared to be swollen over the Achilles' tendon region. Tenderness was elicited over
Received: October 13, 2015

Accepted: March 22, 2016

Accessible online at:

www.onkder.org
Dr. Bilge KESIKBURUN

Dışkapı Yıldırım Beyazıt Eğitim ve Araştırma Hastanesi,

Fiziksel Tıp ve Rehabilitasyon

Ankara-Turkey

E-mail:drbilgekb@gmail.com 

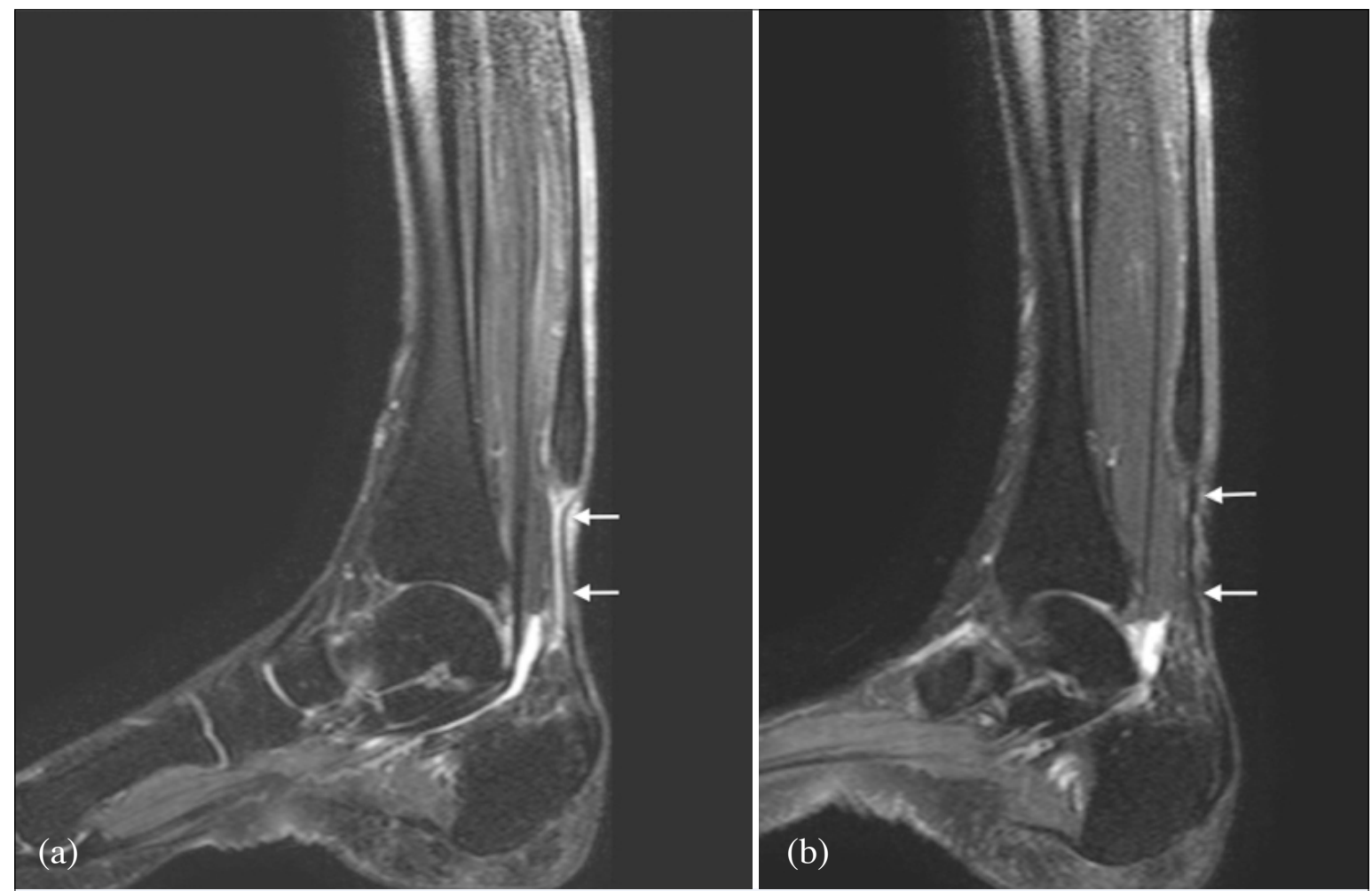

Fig. 1. T2 weighted MRI showing complete tear of (a) the left Achilles' tendon, $9 \mathrm{~cm}$ proximal to the calcaneal insertion and (b) the right Achilles' tendon, $8.5 \mathrm{~cm}$ proximal to the calcaneal insertion.

both Achilles' tendons, near the insertions into the calcaneus. Bilaterally, a gap was determined by palpation of the tendon next to the swollen sites. Thompson's test was positive. Bilaterally, active plantar flexion could only be performed with difficulty. Active and passive plantar flexion of the ankle was $20^{\circ}$ and $30^{\circ}$, respectively. Active and passive dorsiflexion was limited in a neutral position. No neurological deficit was determined. From these findings, a preliminary diagnosis of Achilles' tendon rupture was considered, and magnetic resonance imaging (MRI) revealed a complete tear of the left Achilles' tendon $9 \mathrm{~cm}$ proximal to the calcaneal insertion, and a complete tear of the right Achilles' tendon $8.5 \mathrm{~cm}$ proximal to the calcaneal insertion (Figure 1).

Upon consultation with an orthopedic surgeon, surgical repair was not recommended, due to the delay in the event. The patient undertook a 4-week rehabilitation program of 5 days per week, which included stretchingstrengthening exercises and analgesic physical modalities. Transcutaneous electrical nerve stimulation with a protocol of 20 minutes $/ 100 \mathrm{~Hz} /$ day was administered for pain. Stretching exercises, a cycling program, resistance strengthening with elastic bands, and closed-chain exercises of the gastrocnemius-soleus-Achilles motor unit were performed for 30 minutes a day. The patient was capable of walking with a cane. Pain was assessed with a $10-\mathrm{mm}$ visual analog scale (VAS). At the end of the 4-week rehabilitation program, VAS score had reduced from 6 to 0 , and the patient was able to walk up and down stairs. Active and passive plantar flexion of the ankle was $30^{\circ}$ and $40^{\circ}$, respectively. Active and passive dorsiflexion were $10^{\circ}$, though the patient was still unable to walk on the toes.

Foot and ankle problems were evaluated by the Foot and Ankle Outcome Score (FAOS) at the beginning of therapy. FAOS was developed to assess problems in patients with lateral ankle instability, Achilles' tendonitis, and plantar fasciitis. Reliability and validity studies of the FAOS among the Turkish population have been conducted.[3] The FAOS includes 5 subscales; pain, other symptoms, function in daily living, function in sport and recreation, and foot and ankle-related quality of life. A normalized score (100 indicating no symptoms, 0 indicating extreme symptoms) is calculated for each subscale. In the present patient, the pain subscale of FAOS had improved from 56.6 to 94.5 on 3-month follow-up examination. Informed consent was obtained. 


\section{Discussion}

The Achilles' tendon is the largest and strongest tendon in the human body. It is formed by the combined aponeuroses of the gastrocnemius and soleus muscle tendons and is attached to the middle part of the posterior surface of the calcaneus. The tendon is responsible for plantar flexion of the foot at the ankle joint.[4] Achilles' tendon rupture usually occurs secondary to traumatic injuries with sudden dorsiflexion of the ankle while the knee is extended. Ruptures typically occur 2-6 cm proximal to the tendon insertion into the calcaneus.[5] However, rupture may occur above $6 \mathrm{~cm}$ proximal to tendon insertion in tall patients, as was the case with the present patient, who was $185 \mathrm{~cm}$ in height.

Achilles rupture may also occur spontaneously. Risk factors for spontaneous rupture have been reported as rheumatoid arthritis, diabetes mellitus, organ transplantation, renal failure, gout, systemic lupus erythematosus, and systemic corticosteroid and fluoroquinolone treatment.[6] Spontaneous rupture is a rare occurrence, with a reported incidence of $0.002 \%$ in Western populations, of which only $1 \%$ is simultaneous bilateral rupture. [4,7] Corticosteroid use is responsible for approximately half of reported spontaneous cases.[8]

The mechanism of rupture associated with steroid use is not yet fully understood, though several mechanisms have been suggested. Corticosteroids cause inhibition of fibroblast proliferation and maturation, leading tendon repair process and collagen synthesis to decrease. In addition, degenerative changes occur in the Achilles' tendon following the third decade of life, and the suppressing effect of corticosteroids on healing in a degenerative tendon may lead to tendon rupture as a result of only minor trauma. [9] Chemotherapy agents are generally toxic, and have many adverse effects, such as myelosuppression, and renal, cardiac, and liver toxicity. [10] Previously unknown adverse effects may also occur during treatment. Because chemotherapy agents may damage musculoskeletal structures, chemotherapeutics other than steroids may have additional impact by decreasing tendon quality, as in the present case. However, to the best of our knowledge, no case of tendon rupture associated with chemotherapy agents other than steroids has been reported.

Diagnosis of Achilles' tendon rupture is usually clinical, relying upon the presence of a palpable gap in the tendon and bulging in the muscle. MRI and ultrasonography are used to aid in confirmation of diagnosis.

Open surgery, percutaneous surgery or conservative methods may be used in the treatment of Achilles' ten- don rupture. Conservative treatment typically includes immobilization of the ankle joint in the equinus position, a semi-equinus position, and a neutral position. Immobilization is provided by cast or cast-boot, though duration of knee cast use has yet to be clarified.[11] Treatment selection depends upon the patient's age, time of injury, comorbidity, and patient expectations. [12] Surgical treatment is usually preferred in the early period and for younger, active, or athletic individuals. [13] Conservative treatment was selected in the present case, as the patient was older, and the tear was chronic.

In conclusion, chemotherapy agents can cause many complications. Diagnosis of Achilles rupture may be challenging during chemotherapy protocol. Spontaneous rupture of the Achilles' tendon induced by corticosteroid use is a rare complication. However, it should be considered in cases of patients who are taking chemotherapy agents including steroids.

\section{Disclosure Statement}

The authors declare no conflicts of interest.

\section{References}

1. Ilan DI, Tejwani N, Keschner M, Leibman M. Quadriceps tendon rupture. J Am Acad Orthop Surg 2003;11(3):192-200.

2. Kotnis RA, Halstead JC, Hormbrey PJ. Atraumatic bilateral Achilles tendon rupture: an association of systemic steroid treatment. J Accid Emerg Med 1999;16:378-9.

3. Karatepe AG, Günaydin R, Kaya T, Karlibaş U, Özbek G. Validation of the Turkish version of the foot and ankle outcome score. Rheumatol Int 2009;30:169-73.

4. Khanzada Z, Rethnam U, Widdowson D, Mirza A. Bilateral spontaneous non-traumatic rupture of the Achilles tendon: a case report. J Med Case Rep 2011;5:263.

5. DiStefano VJ, Nixon JE. Achilles tendon rupture: pathogenesis, diagnosis, and treatment by a modified pullout wire technique. J Trauma 1972;12(8):671-7.

6. Price AE, Evanski PM, Waugh TR. Bilateral simultaneous achilles tendon ruptures. A case report and review of the literature. Clin Orthop Relat Res 1986;(213):249-50.

7. Kelly M, Dodds M, Huntley JS, Robinson CM. Bilateral concurrent rupture of the Achilles tendon in the absence of risk factors. Hosp Med 2004;65(5):310-1.

8. Blanco I, Krähenbühl S, Schlienger RG. Corticosteroid-associated tendinopathies: an analysis of the pub- 
lished literature and spontaneous pharmacovigilance data. Drug Saf 2005;28(7):633-43.

9. Hayes T, McClelland D, Maffulli N. Metasynchronous bilateral Achilles tendon rupture. Bull Hosp Jt Dis 2003;61(3-4):140-4.

10. Doru T. Chemotherapy toxicities and complications. In: Clinical Hematology, Philadelphia: Elsevier-Mosby; 2006. p. 1144-54.

11. Osarumwense D, Wright J, Gardner K, James L. Conservative treatment for acute Achilles tendon rupture: survey of current practice. J Orthop Surg (Hong Kong)
2013;21(1):44,6.

12. Metzl JA, Ahmad CS, Levine WN. The ruptured Achilles tendon: operative and non-operative treatment options. Curr Rev Musculoskelet Med 2008;1(2):1614.

13. Lansdaal JR, Goslings JC, Reichart M, Govaert GA, van Scherpenzeel KM, Haverlag R, et al. The results of 163 Achilles tendon ruptures treated by a minimally invasive surgical technique and functional aftertreatment. Injury 2007;38(7):839-44. 